We confirmed expression of eNOS and beta-catenin in both UF and DF zones by quantitative-PCR and immunostaining, as well as the interaction between eNOS and beta-catenin in both regions by proximity ligation assay. We then studied the expression of several pro-survival and anti-apoptotic genes by q-PCR in HUVEC exposed to flow for 72 hours. We observed that the expression of $\mathrm{Bcl}-2$ and survivin were downregulated in UF exposed cells compared to static conditions $(62 \%$ \pm 0.08 downregulation of $\mathrm{Bcl}-2 \%$ and $67 \% \pm 0.08$ downregulaation of survivin, $n=3 \quad p<0.01$ in both cases); and using specific beta-catenin/TCF-LEF inhibitors we identified survivin as an anti-apoptotic gene regulated by beta-catenin $(89 \%$ \pm 0.03 downregulation in inhibitor treated samples compared to non-treated, $\mathrm{n}=3 \mathrm{p}<0.001)$ in endothelial cells under flow.

We also investigated the reciprocal effects on eNOS of activation of Wnt signalling and beta-catenin in HUVECs. Using Wnt3a and $\mathrm{LiCl}$, that lead to the accumulation of beta-catenin in the cytoplasm, we found that phosphorylation of eNOS at Ser1177 increased 4 fold after $2-5 \min (n=3 p<0.01)$ leading to enzyme activation. Phosphorylation of eNOS at Ser633 and Ser114 was also observed both in HUVEC exposed to UF flow for 72 hour in an orbital shaker and in HUVEC stimulated by Wnt3a.

Together our results indicate that beta-catenin is a key mediator of flow-induced anti-apoptotic effects, both through transcriptional regulation and through activation of eNOS phosphorylation in endothelial cells.

This work has been supported by a grant from the British Heart Foundation.

\section{INSULIN-LIKE GROWTH FACTOR BINDING PROTEIN 2 (IGFBP2): A POSITIVE REGULATOR OF ANGIOGENESIS?}

Alexander-Francisco Bruns*, Jessica Smith, Nadira Yuldasheva, Mark T Kearney, Stephen B Wheatcroft. University of Leeds

\subsection{6/heartjnl-2017-311726.171}

Introduction The insulin-like growth factor binding protein 2 (IGFBP2) has been implicated in the regulation of insulin-like growth factor (IGF) activity in most tissue and organs. IGFBP2 has, however, been reported to have additional intrinsic, IGF independent properties. Low circulating IGFBP2 levels are associated with obesity in humans. High levels of IGFBP2 on the other hand are linked to increased tumour angiogenesis in humans. In this setting increased angiogenesis has been suggested to be caused by indirect rather than direct modulation of endothelial cells. Here we tested the hypothesis that IGFBP2 is able to modulate endothelial cell function directly. Basic methods and Results: Using immunoblotting, we show that acute stimulation of human umbilical vein endothelial cells (HUVEC) with $15 \mathrm{nM}$ IGFBP2 lead to an increase in phosphorylation of $\mathrm{Akt} / \mathrm{PKB}$, an important regulator of endothelial cell function. Data obtained from an in vitro model of sprouting angiogenesis suggests that stimulation of HUVEC with IGFBP2 induced endothelial cell sprouting. Mice overexpressing human IGFBP2 showed increased tip cell formation and vascular density in the mouse retina model of developmental angiogenesis.

Conclusions Here we present data from in vitro and in vivo models of angiogenesis supporting our hypothesis that IGFBP2 is able to directly modulate endothelial cell function.

\section{COMPARISON OF THE MECHANICAL PERFORMANCE OF POLYMERIC AND METALLIC SCAFFOLDS - TESTING AND MODELLING}

${ }^{1}$ Raasti Naseem*, ${ }^{2}$ Vadim Silberschmidt, ${ }^{2}$ Yang Liu, ${ }^{3}$ Syed Hossainy, ${ }^{3}$ Senthil Eswaran, ${ }^{3}$ Chad Abunassar, ${ }^{2}$ Liguo Zhao. 'Lougborough University; ${ }^{2}$ Loughborough University; ${ }^{3}$ Abbott Vascular

10.1136/heartjnl-2017-311726.172

Percutaneous coronary intervention is a standard procedure to resolve blockages within artery, which involves the implantation of stents to maintain vessel patency. Currently, bioresorbable scaffolds (BRSs) are in the process of replacing the metallic permanent predecessor (drug eluting stents) commonly used in stenting. BRSs are commonly made of poly (L) Lactide (PLLA), an aliphatic polyester which is biodegradable and biocompatible with a wide range of medical applications. The performance of these scaffolds is not well defined in comparison to their metallic counterparts.

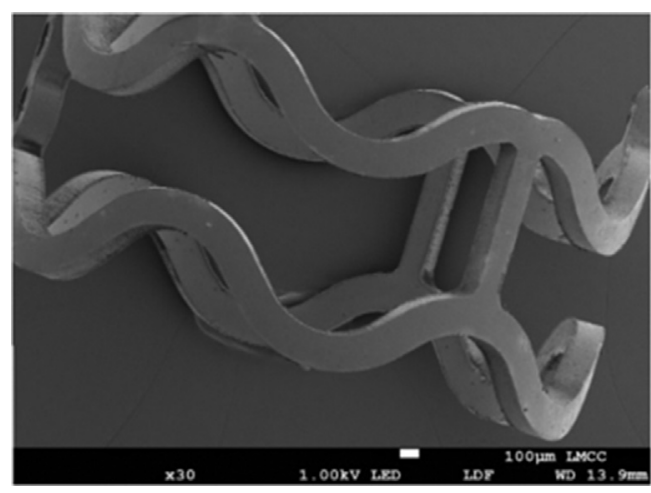

Abstract 174 Figure 1 SEM image of a polymeric scaffold.

The aim of this project is to assess the mechanical performance of PLLA scaffolds (Figure 1), with a direct comparison to that of metallic stents. This will be achieved through mechanical testing of structural rings at different load rates and ranges. Scaffolds will also be characterised using nano/ micro indentation. The results will be used to support computational work for predicting the behaviour of both stents during crimping and expansion (Figure 2).
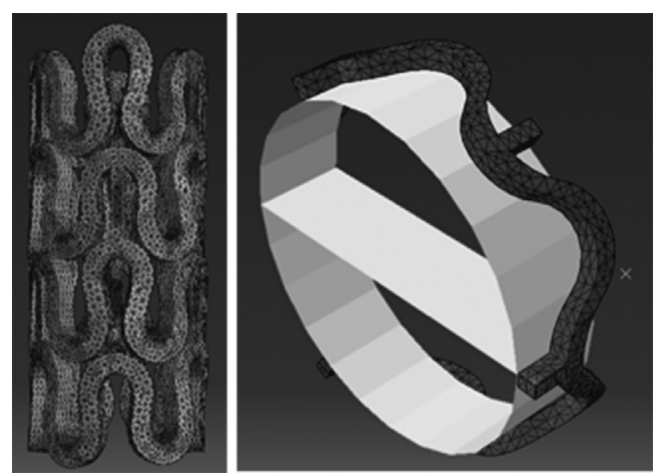

Abstract 174 Figure 2 Illustration of computational assessments of BRS scaffold performance (ring test and crimping).

Figure 3, Nanoindentation data on BRS.

Figure 4, AFM data on BRS. 